\title{
The role and possible mechanism of the long noncoding RNA LINC01260 in nonalcoholic fatty liver disease
}

Xiaoxiao Ge ${ }^{1,4 \dagger}$, Tao Sun ${ }^{1,2 \dagger}$, Yanmei Zhang ${ }^{1,5}$, Yongqing Li ${ }^{1}$, Peng Gao ${ }^{3}$, Dantong Zhang ${ }^{2}$, Bingyang Zhang ${ }^{1,2}$, Peijun Wang ${ }^{1}$, Wanshan $\mathrm{Ma}^{1,2^{*}}$ and Sumei $\mathrm{Lu}^{1,2^{*}}$

\begin{abstract}
Objective: To investigate the differential expression profile of IncRNAs in the nonalcoholic fatty liver disease (NAFLD) model induced by oleic acid (OA) and to further explore the role of LINC01260 (ENST00000255183) in NAFLD, providing theoretical support for the clinical value of IncRNAs in NAFLD.

Methods: $\mathrm{OA}(50 \mu \mathrm{g} / \mathrm{mL})$ was used to induce steatosis in normal human LO2 hepatocytes for $48 \mathrm{~h}$ and was verified by Oil red $O$ staining. Differential expression profiles of IncRNAs were obtained by eukaryotic circular sequencing (RNA/IncRNA/circRNA-seq) techniques. A gain-of-function (GOF) strategy for LINC01260 was adopted, Oil red O staining and semiquantitative analysis were combined to explore whether the GOF of LINC01260 affects LO2 cell steatosis. CeRNA-based bioinformatics analysis of IncRNAs was performed, and the enriched mRNAs were further verified. RXRB siRNAs were applied and verify its role in LINC01260 regulated OA-induced hepatocytes steatosis.
\end{abstract}

Results: Lipid droplets of different sizes were observed in the cells of the OA group. Absorbance in the OA group was significantly increased after isopropanol decolorization $(P<0.05)$. Compared with those in the control group, there were 648 IncRNAs with differential expression greater than 1 time in the OA group, of which 351 were upregulated and 297 were downregulated. Fluorescence quantitative PCR showed that the expression of LINC01260 in the OA group was downregulated by $0.35 \pm 0.07$-fold $(P<0.05)$. The formation of lipid droplets in LO2 cells of the LINC01260 GOF group decreased significantly $(P<0.05)$. CeRNA analysis indicated that the mRNA levels of RXRB, RNPEPL1, CD82, MADD and KLC2 were changed to different degrees. Overexpression of LINC01260 significantly induced RXRB transcription $(P<0.05)$ and translation, and RXRB silence attenuated the lipids decrease induced by LINC01260 overexpression.

Conclusion: The OA-induced NAFLD cell model has a wide range of IncRNA differential expression profiles. LINC01260 participates in the regulation of the lipid droplet formation process of NAFLD, and its overexpression can significantly inhibit the steatosis process of LO2 cells. Mechanistically, LINC01260 may act as a ceRNA to regulate the expression of RXRB, thereby affecting the adipocytokine signaling pathway.

\footnotetext{
*Correspondence: mwsqianyi@163.com; Ismqianyi@126.com

${ }^{\dagger}$ Xiaoxiao Ge and Tao Sun have contributed equally.

1 Department of Laboratory Medicine, Shandong Provincial Qianfoshan

Hospital, Shandong University, Jinan 250014, Shandong, People's Republic of China

Full list of author information is available at the end of the article
} permits use, sharing, adaptation, distribution and reproduction in any medium or format, as long as you give appropriate credit to the original author(s) and the source, provide a link to the Creative Commons licence, and indicate if changes were made. The images or other third party material in this article are included in the article's Creative Commons licence, unless indicated otherwise in a credit line to the material. If material is not included in the article's Creative Commons licence and your intended use is not permitted by statutory regulation or exceeds the permitted use, you will need to obtain permission directly from the copyright holder. To view a copy of this licence, visit http://creativecommons.org/licenses/by/4.0/. The Creative Commons Public Domain Dedication waiver (http://creativeco mmons.org/publicdomain/zero/1.0/) applies to the data made available in this article, unless otherwise stated in a credit line to the data. 
Keywords: Nonalcoholic fatty liver disease, Long-chain noncoding RNAs, Eukaryotic circular sequencing, Oleic acid, Hepatocyte steatosis

\section{Introduction}

Nonalcoholic fatty liver disease (NAFLD) is a syndrome mainly characterized by diffuse hepatocyte bullous fat. The cause of NAFLD is very complicated, but alcohol and other clear liver damage factors are excluded [1]. NAFLD has become the most common chronic liver disease and the primary reason for abnormal liver biochemical indicators in health examinations in China [2], bringing a considerable health and economic burden to patients, families, and society.

Long noncoding RNAs (lncRNAs) are noncoding RNAs with lengths greater than 200 base pairs. Because of the lack of an open reading frame, lncRNAs do not encode proteins. LncRNAs can regulate many biological processes, including the cell cycle, apoptosis, and differentiation [3]. In recent years, many studies have confirmed that lncRNAs play an important role in the occurrence and development of NAFLD [4-7]. Emerging evidence suggests that lncRNAs may regulate hepatic lipid metabolism in NAFLD.

The most well-known lncRNA is H19, which is highly expressed in NAFLD. Previous studies showed that H19, as a miRNA decoy, targets miR-130a to regulate PPARy expression and plays an important role in NAFLD [8]. Wang investigated the role of H19 in hepatic lipid metabolism and its potential association with NAFLD [9]. They found that exogenous overexpression of H19 in hepatocytes induced lipid accumulation and upregulated the expression of numerous genes involved in lipid synthesis, storage and breakdown. Mechanistically, H19 induces hepatic steatosis by activating the MLXIPL and mTORC1 networks in hepatocytes. In addition to H19, several other lncRNAs are involved in NAFLD pathogenesis, particularly in liver steatosis. Another lncRNA is Gm15622, which stimulates SREBP-1c expression and hepatic lipid accumulation by sponging miR-742-3p in mice [10]. Additionally, the lncRNA Gm12664-001 was demonstrated to attenuate hepatic lipid accumulation by negatively regulating miR-295-5p and enhancing CAV1 expression in AML12 cells [11]. In addition, the IncRNA Gomafu upregulates the expression of the transcription factor FoxO1 by sponging miR-139-5p, thereby promoting insulin resistance in the liver, reducing the lipolysis ability of the liver, and increasing lipid deposition in NAFLD [12]. However, the mechanism of action of lncRNAs in NAFLD has not yet been fully elucidated. Identifying additional novel lncRNAs involved in NAFLD is still important.
Competing endogenous RNAs (CeRNA) hypothesis RNA transcripts can crosstalk by competing for common microRNAs, with microRNA response elements (MREs) as the foundation of this interaction [13]. These RNA transcripts have been termed as competing endogenous RNAs-ceRNAs [14]. Any RNA transcript with MREs might act as ceRNA, and ceRNAs include pseudogene transcripts, lncRNAs, circRNAs and mRNAs, these transcripts can compete for the same microRNA response elements (MERs) to regulate mutually. To find potential target of microRNAs, the target/microRNAs is usually predicted with home-made miRNA target prediction software based on TargetScan \& miRanda [15, 16].

In this article, we established lncRNA profile in an in vitro model of NAFLD in cultured LO2 cells using oleic acid (OA) stimulation. Six lncRNAs were selected to explore their roles in NAFLD. Then, LINC01260 (ENST00000255183) was investigated in detail, and its potential mechanism as a ceRNA in NAFLD was investigated. Finally, LINC01260 was proposed as a key hepatic steatosis regulator, and its mechanism is probably related to the upregulation of RXRB expression as ceRNA. The present study provides theoretical support for the clinical value of LINC01260 in NAFLD.

\section{Materials and methods Reagents}

The normal human liver cell line LO2 (HL-7702) was purchased from Wuhan Procell Life Science \& Technology Co., Ltd. (China). An RNA extraction kit was obtained from Shanghai Yishan Biotechnology Co., Ltd. (China). A RevertAid First Strand cDNA Synthesis Kit (\#k1622) was purchased from Thermo Fisher Scientific (USA). FastStart Essential DNA Green Master Mix was obtained from Roche (Switzerland). Lipofectamine 2000 transfection reagent was purchased from Invitrogen (USA); the IncRNA overexpression plasmid, negative control plasmid, and lncRNA primers were designed and synthesized by Biosune Biotechnology (Shanghai) Co., Ltd. (China).

\section{Cell culture and hepatic steatosis model induction}

LO2 cells were cultured in RPMI-1640 medium supplemented with $10 \% \mathrm{FBS}, 100$ units $/ \mathrm{mL}$ penicillin, and $100 \mu \mathrm{g} / \mathrm{mL}$ streptomycin. When the cell density reached approximately $60 \%$, LO2 cells were exposed to different doses of OA $(10,20,30,40,50,60 \mu \mathrm{g} / \mathrm{mL})$ or DMSO for $48 \mathrm{~h}$. The medium were changed every $24 \mathrm{~h}$. 


\section{Oil red $O$ staining}

After steatosis induction, LO2 cells were fixed with $4 \%$ tissue/cell fixative for $10 \mathrm{~min}$ and then stained for 30 min with Oil red $\mathrm{O}(0.6 \mathrm{mg} / \mathrm{mL})$ under room temperature and dark conditions. After staining, 60\% isopropanol was added for 1 min to remove the background signal. An inverted microscope (Olympus, Japan) was used to observe droplets in cells. Then, decolorization was performed using $100 \%$ isopropanol for $10 \mathrm{~min}$ at $37^{\circ} \mathrm{C}$. The OD value of the decolorization solution was detected by a microplate reader at a wavelength of $510 \mathrm{~nm}$.

\section{LncRNA sequencing}

Eight samples were collected consisting of 4 control and 4 experimental samples, labeled control (ctrl) 1 , $\operatorname{ctrl} 2$, $\operatorname{ctrl} 3, \operatorname{ctrl} 4$, test 1 , test 2 , test 3 , and test 4 . Control group means LO2 cells exposed to DMSO for $48 \mathrm{~h}$; test group means LO2 cells exposed to OA $(50 \mu \mathrm{g} / \mathrm{mL})$ for $48 \mathrm{~h}$. Then, cells were harvested in Trizol reagent and all samples were sent to Shanghai Kangcheng Biotechnology Co., Ltd. for eukaryotic circular sequencing to detect the expression changes in lncRNAs in the NAFLD cell model. The general protocol, including cell treatment, RNA extraction, RNA library establishment, qPCR quantification and sequencing, was described in published work (Lipids Health Dis. 2021; 20(1):39).

\section{Quantitative real-time PCR (qPCR)}

An RNA extraction kit was used to extract total RNA as protocol. Microarray data were validated by qPCR, with $\beta$-actin as a control. The primer sequences are detailed in Additional file 1: Table S1.

\section{Cell transfection}

Overexpression plasmids of target lncRNAs were constructed with pcDNA3.1 as the vector. Lipofectamine 2000 was the transfection reagent. The detailed steps were performed according to the manufacturer's protocols. Cells were harvested $24 \mathrm{~h}$ after transfection for the luciferase assay. The expression of target lncRNAs was detected using qPCR. All transfection experiments were performed in triplicate.

\section{CeRNA network prediction for the IncRNA LINC01260}

Further bioinformatics analysis was conducted on the lncRNA LINC01260 to construct a ceRNA network and predict its downstream mRNAs. The intersection between the identified downstream mRNAs and an mRNAs profile based on LO2 sequencing results (Lipids Health Dis. 2021; 20(1):39) was performed using the https://bioinfogp.cnb.csic.es/tools/venny/ website. Generally, to find potential target of microRNAs, the target/microRNAs is predicted with homemade miRNA target prediction software based on TargetScan \& miRanda. Besides a measure with the number of common microRNAs, a hypergeometric test is executed for each ceRNA pair separately. In the present study, we first get mRNAs intersection based on bioinformatics mRNA prediction data and mRNA profile from RNA Sequencing. Then, target miRNAs were predicted for the intersection mRNAs. Then, qRT-PCR verification was performed to verify the resulting mRNAs.

\section{RXRB siRNAs sequences}

Three specific siRNAs for RXRB were designed, and they are RXRB siRNA-1187, siRNA-1311, siRNA-1512. Meaningless control sequences were synthesized as negative control. These siRNAs were transfected into LO-2 cells, respectively, to investigate the effect of RXRB silence to OAinduced steatosis. The siRNAs sequences were as follows:

siRNA-1187: Sense (5'-3')-GACCCUGUGACUAAC AUCUTT

Antisense (5'-3')-AGAUGUUAGUCA CAGGGUCTT

siRNA-1311: Sense (5'-3')-GCUGGAAUGAACUCC UCAUTT

Antisense (5'-3')-AUGAGGAGUUCA UUCCAGCTT

siRNA-1512: Sense (5'-3')-GGGCAAUCAUUCUGU UUAATT

Antisense (5'-3')-UUAAACAGAAUG AUUGCCCTT

\section{Statistical analysis}

All data in this study are presented as the mean \pm SD, and SPSS 19.0 software was used for the statistical analysis. Differences between two groups were compared using Student's $t$-test. Analysis of variance (ANOVA) was used to compare the data of three or more groups. The $q$ test was used for further pairwise comparisons. A value of $P<0.05$ was considered statistically significant.

\section{Results}

A hepatic steatosis model was established in cultured LO2 cells

A hepatic steatosis model was established using cultured LO2 cells by OA induction. Different doses of OA $(10,20$, $30,40,50,60 \mu \mathrm{g} / \mathrm{mL}$ ) were tested, and Oil red O staining showed considerable deposition of lipid droplets in the cells of the OA group compared with the control 
group in an OA dose-dependent manner (Fig. 1a). Moreover, absorbance at $510 \mathrm{~nm}$ in the OA group increased significantly after isopropanol decolorization, indicating increased hepatic lipid accumulation (Fig. 1b). Cell viability of the OA group treated with a dose was less than $50 \mu \mathrm{g} / \mathrm{mL}$ showed no statistically significant decrease compared with the control group (Fig. 1c), indicating that $50 \mu \mathrm{g} / \mathrm{mL}$ OA treatment is the best dose for hepatic steatosis induction.

\section{LncRNA profile changes in a hepatic steatosis model of cultured LO2 cells}

Using eukaryotic circular sequencing techniques, we detected the expression profiles of lncRNAs. The general profile information is shown in Fig. 2. The scatter plot shows that 297 lncRNAs were downregulated and 351 lncRNAs were upregulated in the LO2 steatosis model (Fig. 2a). Then, a karyogram depicting the genomic localization of the host genes of lncRNAs was generated. Further analysis revealed that the upregulated and downregulated lncRNAs were distributed on all chromosomes with the exception of chromosome Y. Chromosomes 1, 5 and 11 contained more lncRNAs than the other chromosomes (Fig. 2b).

\section{General informations of top 20 upregulated and top 20 downregulated IncRNAs}

The top 20 upregulated and top 20 downregulated lncRNAs are scanned. Figure 3 showed the heatmap of the scanned top 20 upregulated and top 20 downregulated lncRNAs. All the fold changes of scanned lncRNAs were higher than twofold, with $P$ valus less than 0.05 . General informations of these lncRNAs were listed in Table 1, including lncRNA_id, associated_gene_name, $\log _{2} \mathrm{FC}$, fold change, and $p$ value.

\section{Overexpression of LINC01260 inhibited hepatic steatosis}

Based on the fold change and $p$ value, six intergenic lncRNAs of interest, namely, lncRNA ENST00000414790, lncRNA ENST00000431095, lncRNA ENST00000608018, lncRNA ENST00000442037, lncRNA ENST00000611525 and LINC01260, were selected for qPCR verification. The

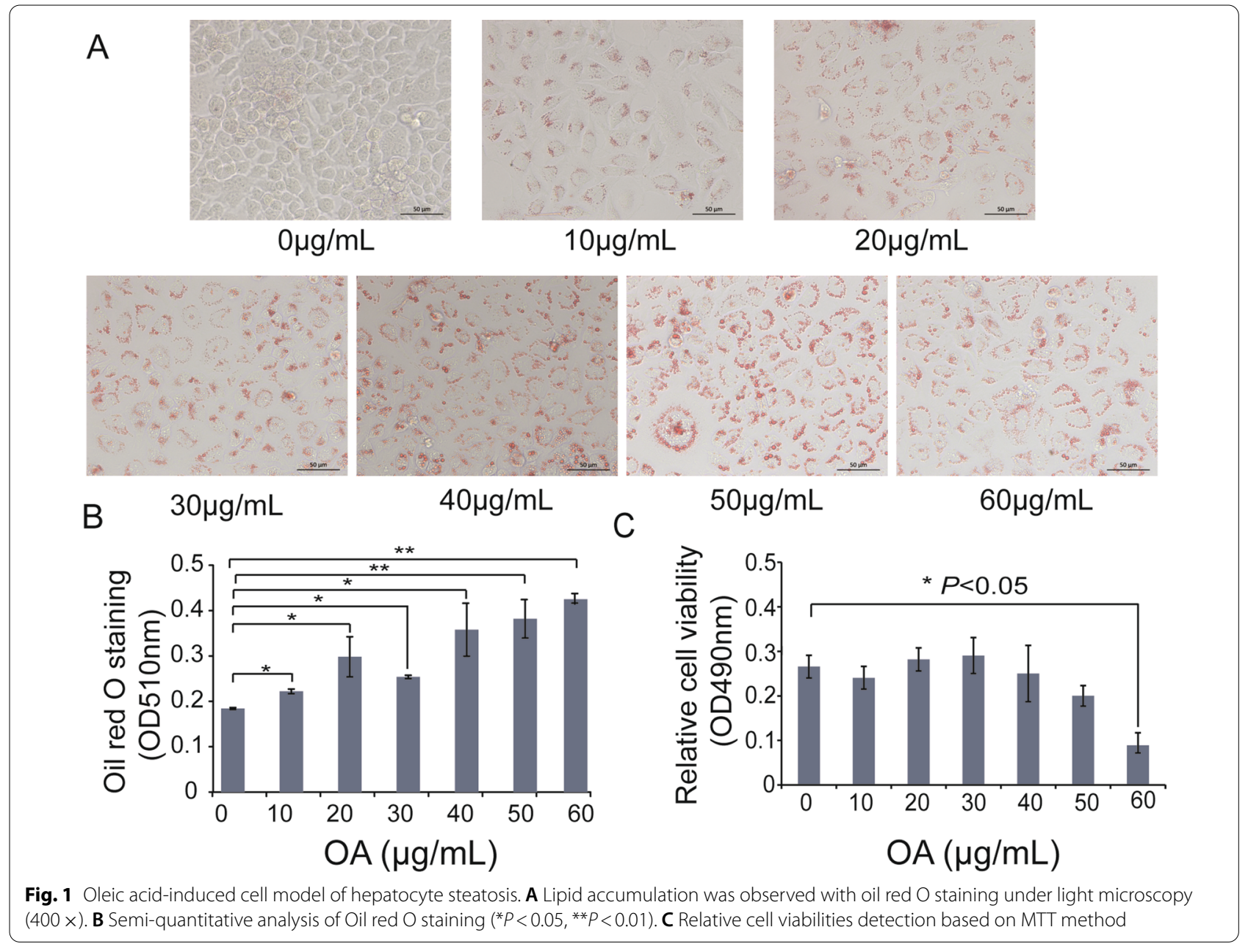




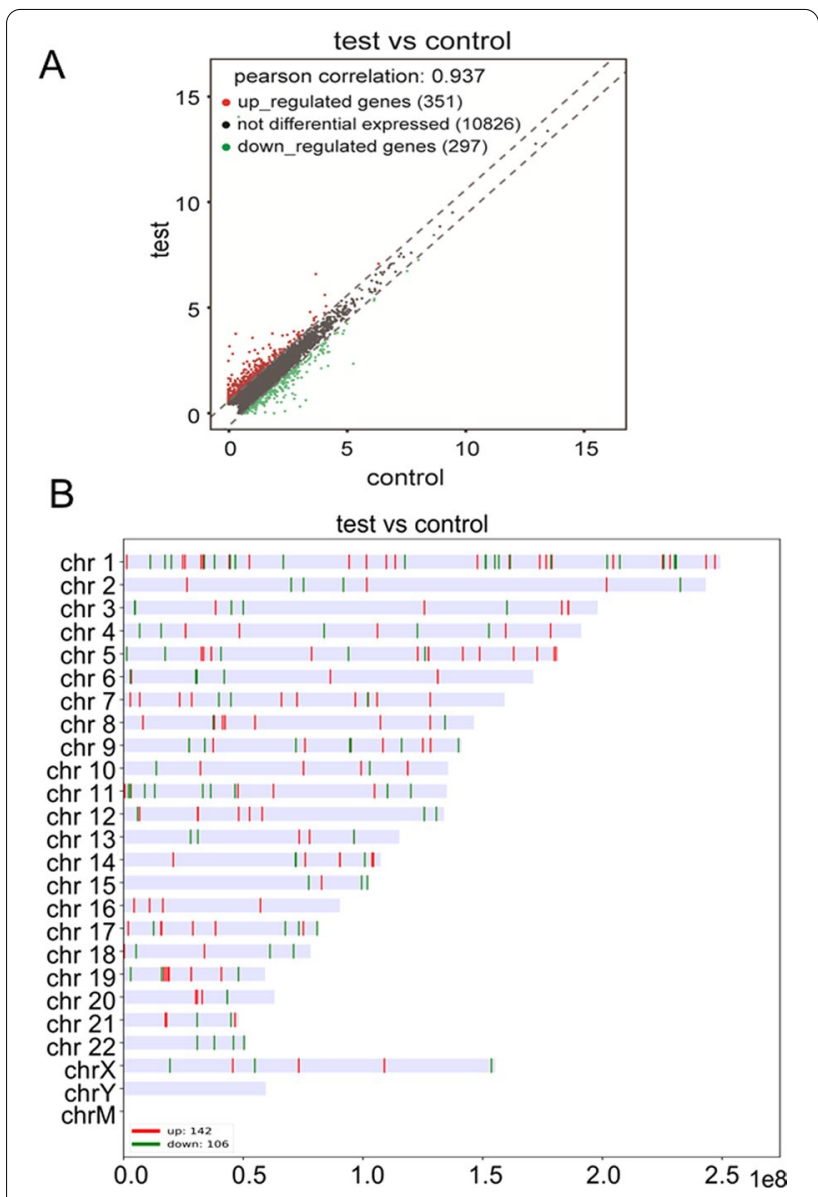

Fig. 2 Profiles of IncRNAs and Karyogram depicting genomic localization of the genes in NAFLD cell model. A The scatter plot showed general profiles changes in IncRNAs between hepatocyte steatosis model and control cells. B Karyogram depicting genomic localization of the genes from co-expressed gene set

results showed that LINC01260, ENST00000442037, and ENST00000611525 were downregulated by $0.35 \pm 0.07$, $0.52 \pm 0.13$, and $0.38 \pm 0.15$, respectively, compared to their expression in the control group (Fig. 4a), which was consistent with the sequencing results $(" P<0.05$, $* * P<0.01)$

Next, we overexpressed the indicated three lncRNAs to investigate whether they could affect hepatocyte steatosis. Fluorescence microscopy showed that the transfection efficiency of the plasmids was approximately $80 \%$ (Fig. 4b). In comparison with those in the control, the levels of LINC01260, lncRNA ENST00000442037, and lncRNA ENST00000611525 in LO2 cells transfected with the overexpression plasmids were increased by $(4151.36 \pm 1316.90) \%,(8946.66 \pm 2405.24) \%$, and $(1605.99 \pm 329.68) \%$, respectively (Fig. 4b). Therefore, these expression plasmids were effective and were used for further experiments.
Then, Oil red $\mathrm{O}$ staining was used to examine lipid accumulation. As shown in Fig. 4c, lipid droplet accumulation did not show much difference in LO2 cells treated with ENST00000442037+ and ENST00000611525+, while lipid droplet accumulation was reduced in LINC01260 + cells compared with pcDNA3.1 cells. Semiquantitative analysis after isopropanol decolorization showed a significant decrease between pcDNA3.1 vector and LINC01260 + groups under OA treatment (Fig. 4d) $(P<0.05)$. Compared with non-OA treated group $(0.22 \pm 0.028)$, OA treatment induced hepatocytes steatosis, with Oil red $\mathrm{O}$ absorbance value increased significantly $(0.51 \pm 0.02)$. Compared with non-treated vector negative control group $(0.22 \pm 0.023)$, OA treated vector control group showed obvious steatosis, with Oil red $\mathrm{O}$ absorbance value increased significantly $(0.48 \pm 0.013)$. The overexpression of LINC01260 leading to a decrease in Oil red staining $(0.42 \pm 0.01)$. These results indicated that overexpression of LINC01260 could inhibit hepatocyte steatosis.

\section{The LINC01260-RXRB axis may be responsible for hepatic steatosis}

Further bioinformatics analysis of LINC01260 was performed, and mRNAs regulated by this IncRNA acting as a ceRNA were predicted. In the present study, there were 241 mRNAs forming a ceRNA network with LINC01260. We compared the sequencing mRNA expression profile with the 241 mRNAs in the ceRNA network and found that there were 5 mRNAs, namely, RXRB, RNPEPL1, CD82, MADD and KLC2, with significant differences. Further, the ceRNA network showed that LINC01260 could indirectly regulate the expression of these mRNAs by competing with 119 miRNAs (Fig. 5a).

To confirm the sequencing results of these mRNAs, qRT-PCR was conducted and showed that RXRB, RNPEPL1 and CD82 were downregulated by (2.24 \pm 0.35$),(2.55 \pm 1.03)$, and $(3.91 \pm 0.92)$ fold, respectively, while MADD and KLC2 were upregulated by $(3.75 \pm 1.36)$ and $(1.20 \pm 0.19)$ fold, respectively, compared with the control group, which was consistent with the sequencing results (Fig. 5b).

Then, the influence of LINC01260 overexpression (LINC01260+) on RXRB, RNPEPL1, CD82, MADD and KLC2 expression was assessed. The results showed that LINC01260 overexpression significantly increased the mRNA level of RXRB compared to that in the pcDNA3.1 group $(* P<0.05)$, with RNPEPL1, CD82, MADD and KLC2 showing no significant alteration $(P>0.05)$ 


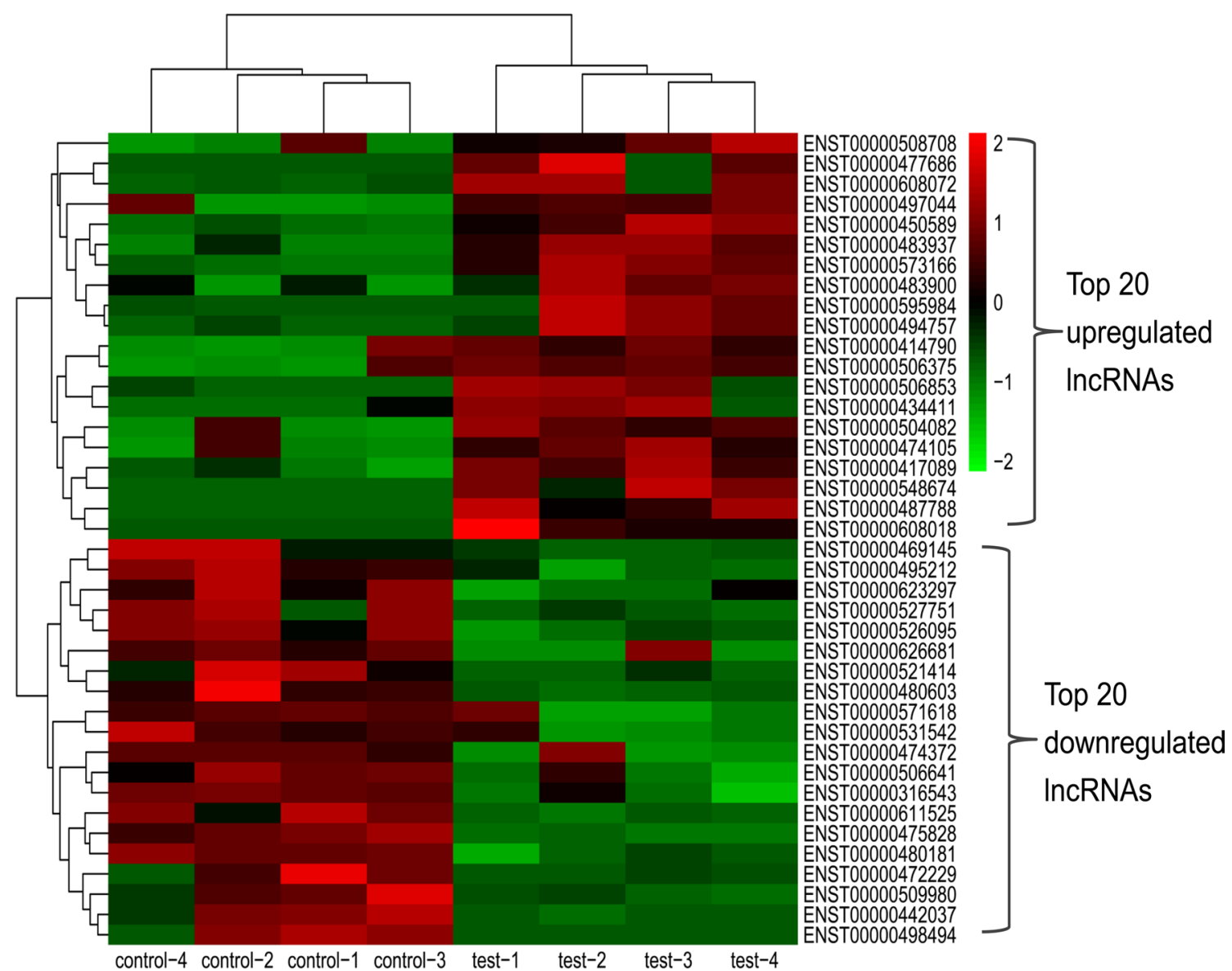

Fig. 3 The heatmap of top 20 upregulated and top 20 downregulated IncRNAs. The top 20 upregulated and top 20 downregulated IncRNAs changed in oleic acid-induced hepatocyte steatosis model were scanned out and heatmap was produced

(Fig. 5c). Western blots detection showed similar unregulated trends of RXRB protein in LINC01260+ cells (Fig. 5d).

Further, RXRB siRNAs effect was identified by qPCR and Western blots, both gene transcription (Fig. 5e) and protein expression (Fig. 5f) showed significant decrease in RXRB. In Oil red O staining, RXRB silence attenuated the decrease of lipids induced by LINC01260 overexpression, with no significant change between RXRB siRNAs group and control group $(P>0.05)$ (Fig. $5 \mathrm{~g})$.

\section{Discussion}

With the improvement of human living standards and changes in dietary structure, the incidence of NAFLD has been increasing yearly. Studies on NAFLD have also received increasing attention. To date, there is no clinically effective drug for the treatment of NAFLD. The main principle of prevention and treatment is to choose liver protection and fat-removing drugs on the basis of diet adjustment and exercise $[17,18]$. Scholars at home and abroad are actively exploring the complex pathogenesis of NAFLD. Considering that the increase in free fatty acids is one of the important mechanisms of NAFLD and that OA has the highest content of free fatty acids, OA was chosen to establish the NAFLD cell model.

Although lncRNAs do not encode proteins, they have important functions in gene expression regulation, such as transcription level regulation, chromosome silencing and modification. At present, studies have found that lncRNAs play an important role in NAFLD and are involved in lipid metabolism, $\beta$-oxidation, IR, inflammation, and liver fibrosis in the process of NAFLD [4-7]. Using lncRNA sequencing technology, researchers analyzed the liver tissues of NAFLD patients and a NAFLD mouse model induced by a high-fat diet $[19,20]$ and found differences in the expression profiles of lncRNAs. To reduce interference factors such as the environment, individual differences and diet in animal NAFLD models and patient specimens, our study applied NAFLD cell models and then detected the differential expression 
Table 1 The details of top 20 up-reguated and down-regulated IncRNAs under oleic acid treatment by RNA sequencing

\begin{tabular}{|c|c|c|c|c|c|c|c|c|c|}
\hline IncRNA_id & $\begin{array}{l}\text { Associated_ } \\
\text { gene_name }\end{array}$ & $\log 2 \mathrm{FC}$ & Fold_Change & p_value & IncRNA_id & $\begin{array}{l}\text { Associated_ } \\
\text { gene_name }\end{array}$ & $\log 2 \mathrm{FC}$ & Fold_Change & $p \_$value \\
\hline Up-reguated & & & & & Down-reguated & & & & \\
\hline ENST00000487788 & MEAF6 & 3.1242 & 8.7195 & 0.0000 & ENST00000442037 & & -2.9296 & 0.131 & 0.0064 \\
\hline ENST00000414790 & & 2.8985 & 7.4566 & 0.0358 & ENST00000472229 & MRPS18B & -2.7050 & 0.1533 & 0.0426 \\
\hline ENST00000497044 & SHTN1 & 2.5531 & 5.8689 & 0.0290 & ENST00000521414 & NDRG1 & -2.6551 & 0.1587 & 0.0076 \\
\hline ENST00000477686 & MRPL20 & 2.4789 & 5.5747 & 0.0261 & ENST00000475828 & MEAF6 & -1.9648 & 0.2561 & 0.0000 \\
\hline ENST00000506375 & SLAIN2 & 2.0151 & 4.0421 & 0.0211 & ENST00000498494 & SELENBP1 & -1.9296 & 0.2625 & 0.0242 \\
\hline ENST00000450589 & ZBTB37 & 1.9146 & 3.7701 & 0.0003 & ENST00000571618 & TBCD & -1.7755 & 0.2921 & 0.0391 \\
\hline ENST00000595984 & COPE & 1.8744 & 3.6666 & 0.0249 & ENST00000509980 & EXOSC9 & -1.7089 & 0.3059 & 0.0094 \\
\hline ENST00000504082 & & 1.7068 & 3.2644 & 0.0198 & ENST00000626681 & $\mathrm{BACH} 1$ & -1.5875 & 0.3327 & 0.0430 \\
\hline ENST00000506853 & AGA & 1.6727 & 3.1882 & 0.0170 & ENST00000469145 & HDGF & -1.3972 & 0.3796 & 0.0148 \\
\hline ENST00000483937 & MAPKAP1 & 1.6650 & 3.1712 & 0.0017 & ENST00000527751 & PRR5L & -1.1769 & 0.4422 & 0.0270 \\
\hline ENST00000483900 & AHCTF1 & 1.6037 & 3.0394 & 0.0486 & ENST00000526095 & KCNQ1 & -1.1363 & 0.4549 & 0.0021 \\
\hline ENST00000474105 & IGF2BP3 & 1.5885 & 3.0074 & 0.0262 & ENST00000611525 & & -1.1224 & 0.4593 & 0.0010 \\
\hline ENST00000417089 & & 1.4379 & 2.7092 & 0.0031 & ENST00000474372 & UBAP2 & -1.0733 & 0.4752 & 0.0484 \\
\hline ENST00000494757 & WIPF2 & 1.4303 & 2.6950 & 0.0122 & ENST00000531542 & AMBRA1 & -1.0705 & 0.4761 & 0.0168 \\
\hline ENST00000608018 & & 1.3741 & 2.5920 & 0.0016 & ENST00000480603 & PPIA & -1.0677 & 0.4770 & 0.0011 \\
\hline ENST00000548674 & ARHGAP9 & 1.3432 & 2.5372 & 0.0019 & ENST00000506641 & CLPTM1L & -1.0613 & 0.4791 & 0.0259 \\
\hline ENST00000434411 & SEC14L1 & 1.3218 & 2.4998 & 0.0455 & ENST00000480181 & CLIC3 & -1.0504 & 0.4828 & 0.0009 \\
\hline ENST00000608072 & $\mathrm{BACH} 1$ & 1.3184 & 2.4939 & 0.0254 & ENST00000495212 & HDGF & -1.0479 & 0.4836 & 0.0042 \\
\hline ENST00000573166 & $\mathrm{HIC1}$ & 1.2892 & 2.4439 & 0.0000 & ENST00000316543 & AACS & -1.0453 & 0.4845 & 0.0105 \\
\hline ENST00000508708 & CSNK1G3 & 1.2802 & 2.4287 & 0.0385 & ENST00000623297 & & -1.0229 & 0.4921 & 0.0142 \\
\hline
\end{tabular}

profiles of lncRNAs in the NAFLD cell models. We identified a total of 648 differentially expressed lncRNAs in the OA group compared to the control group, of which 351 were upregulated and 297 were downregulated. Combined with the results of previous studies, these findings suggest that a variety of lncRNAs are involved in the occurrence and development of NAFLD.

We found that the expression of LINC01260 in the OA group was significantly downregulated, which was consistent with the sequencing results. LINC01260, also known as lncRNA ENST00000255183, is an intergenic lncRNA located on human chromosome 20. This lncRNA is poorly conserved, and its homologous sequence does not exist in mice or rats. At present, there are few studies on LINC01260. Researchers in South Korea conducted a genome-wide copy number variation study on osteoporotic fracture patients and healthy controls and found that one of the significantly related regions is located upstream of LINC01260, which provides an indication of the genetic factors associated with the risk of osteoporotic fractures [21]. Studies have found that patients with melanoma can be divided into high-risk groups and lowrisk groups with different survival times by a molecular signature of six lncRNAs, including LINC01260, HCP5, PIGBOS1, RP11-247L20.4, CTA-292E10.6, and CTB$113 \mathrm{P} 19.5$ [22]. In addition, it has been reported that
LINC01260 expression is downregulated in spinal glioma, and overexpression of LINC01260 targets CARD11 through the NF- $\mathrm{KB}$ signaling pathway to inhibit the proliferation, migration and invasion of spinal glioma cells [23].

There is no report on the role of LINC01260 in NAFLD. To explore the function of LINC01260 and the potential therapeutic value of LINC01260 for treating NAFLD, we constructed a LINC01260 overexpression plasmid. Interestingly, overexpression of LINC01260 significantly inhibited the fatty change process induced by OA in LO2 cells, suggesting that LINC01260 is closely related to lipid accumulation in NAFLD cell models.

Moreover, further competing endogenouse RNA (ceRNA) bioinformatics analysis predicted 5 mRNAs as targets, including RXRB, which was proved positively correlated with LINC01260. We found that RXRB expression was downregulated in NAFLD. RXRB is a member of the nuclear receptor superfamily of retinoic acid $X$ receptors (RXRs) and is expressed in almost all tissues. RXRs have been reported to mediate lipid metabolism by forming dimers with PPAR [24]. Activating RXRs/PPAR $\alpha$ increases the $\beta$-oxidation of fatty acids by upregulating the expression of the fatty acid oxidation rate-limiting enzyme SCAD [25]. Mechanistically, based on in vitro GOF experiments, we determined that overexpression 


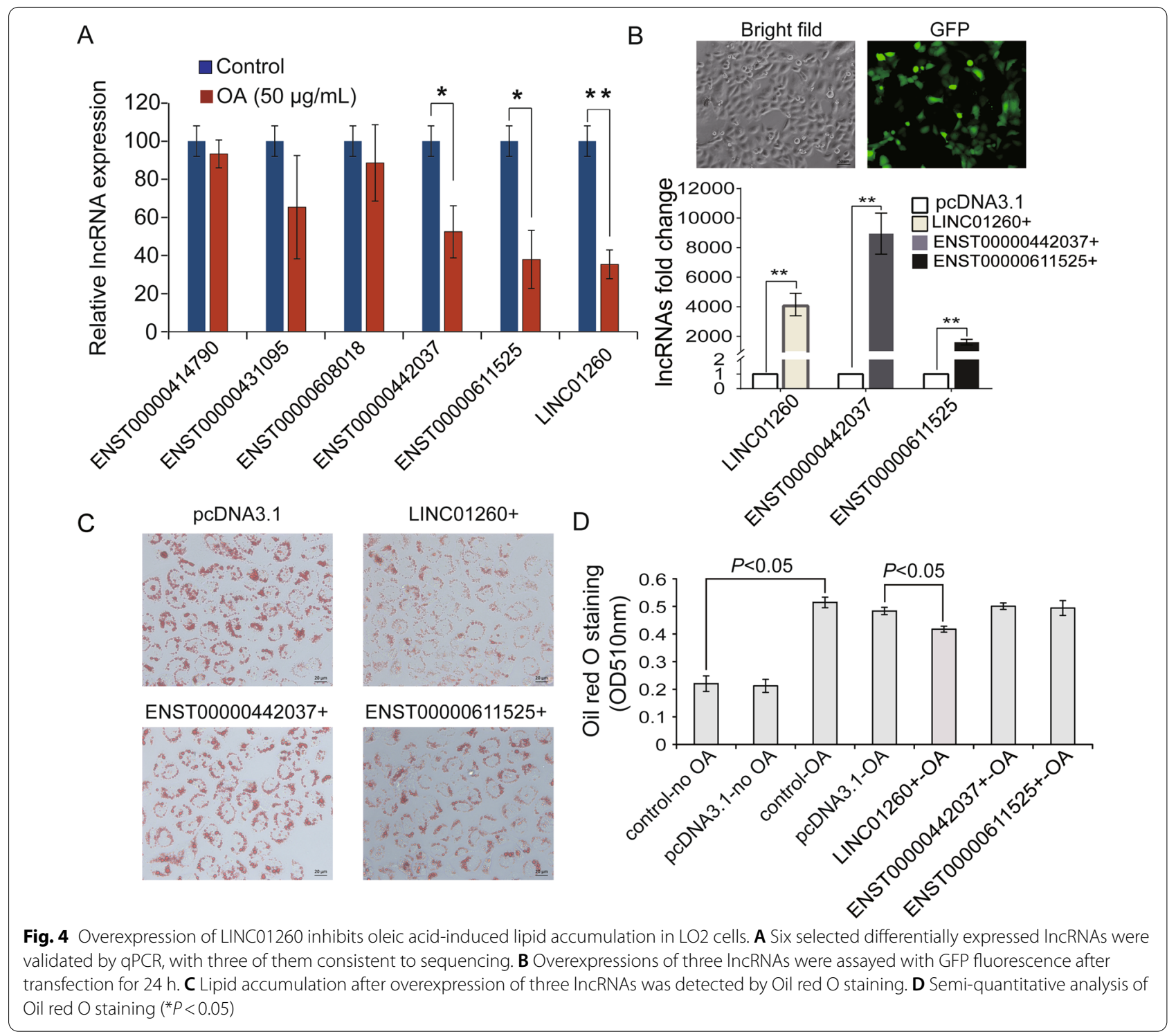

of LINC01260 can positively regulate RXRB expression. Thus, it is conceivable that LINC01260 may regulate hepatic lipid homeostasis through ceRNA mechanism possibly involving the expression of RXRB. In NAFLD, LINC01260 was down regulated, so the miRNA binding with LINC01260 decreased. At the same time, the competing miRNA binding with RXRB increased. Since miRNA negatively regulate target RNA, so RXRB gene expression decreased under LINC01260 decreasing state. Thus, in NAFLD, LINC01260 positively regulate RXRB as ceRNA and regulate NAFLD process.

It should be noted that the fold changes for RXRB gene and protein expression are both small. Less than one fold change in transcription sometimes showed meaningless and no significant biological effect, although the difference showed significance. From transcription to

(See figure on next page.)

Fig. 5 CeRNA network analysis of LINC01260. A Bioinformatic analysis of mRNAs co-expressed with LINC01260. RXRB, RNPEPL1, CD82, MADD and KLC2 were enriched. $\mathbf{B}$ Validation of co-expressed mRNAs by $q P C R$, and all were consistent with sequencing $\left({ }^{*} P<0.05,{ }^{*} P<0.01\right)$. C Overexpression of LINC01260 (LINC01260+) increased the expression RXRB significantly $\left({ }^{*} P<0.05\right)$ compared with pcDNA3.1 groups. D Western blots proved the upregulation trends of RXRB protein in LINC01260 + cells. E RXRB siRNAs decreased RXRB gene expression significantly $\left({ }^{*} P<0.05\right)$. F RXRB siRNAs decreased RXRB protein expression significantly ( $\left.{ }^{*} P<0.05\right)$. G RXRB siRNA attenuated the decrease of lipids induced by LINC01260 overexpression 

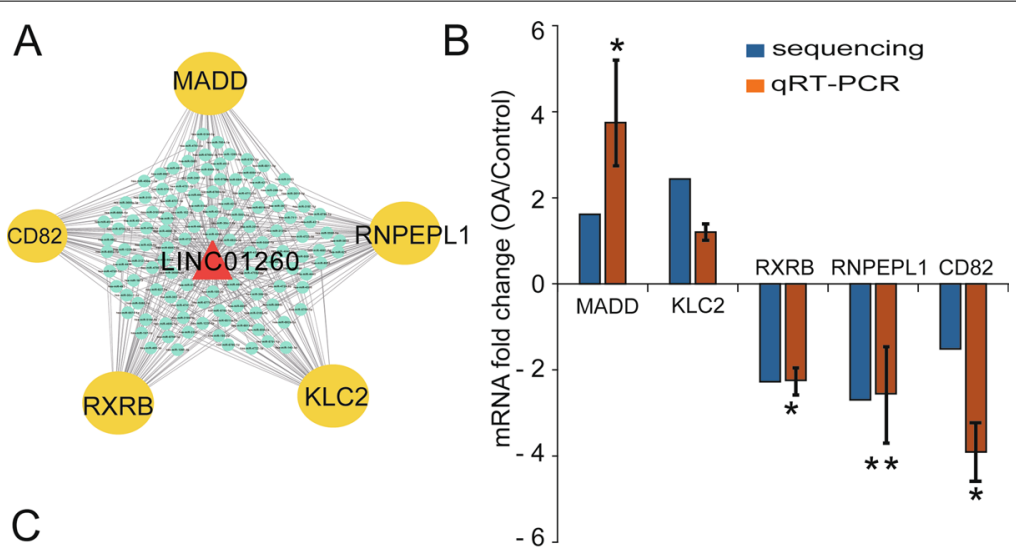

C
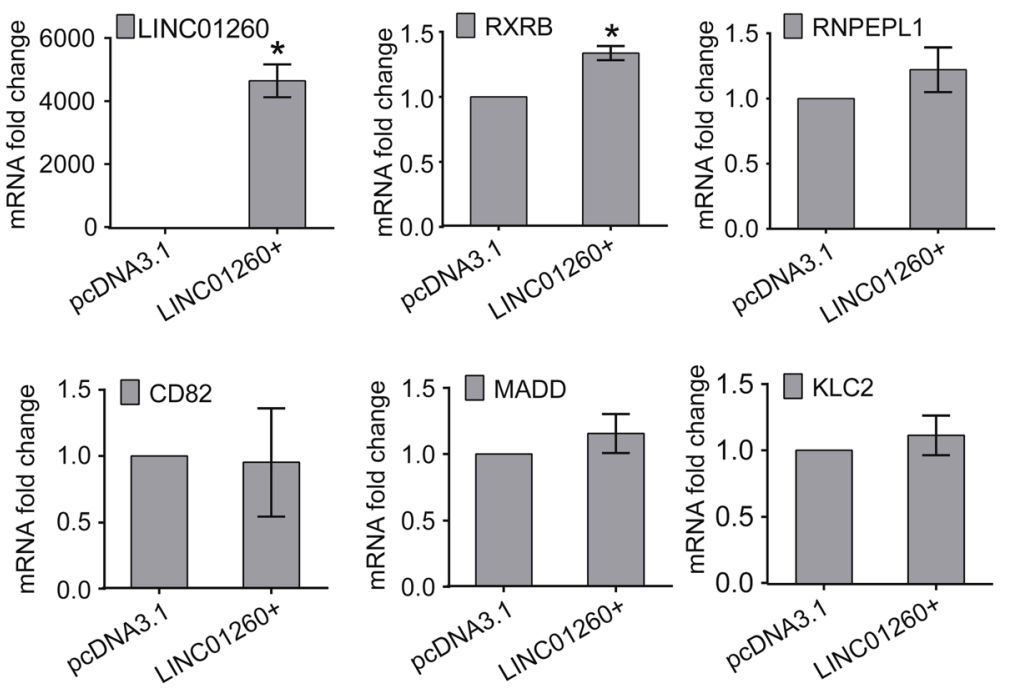

D

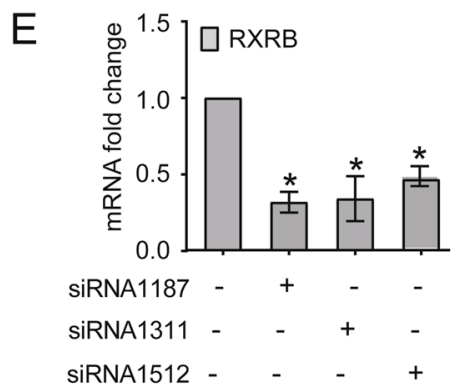

$\mathrm{F}$

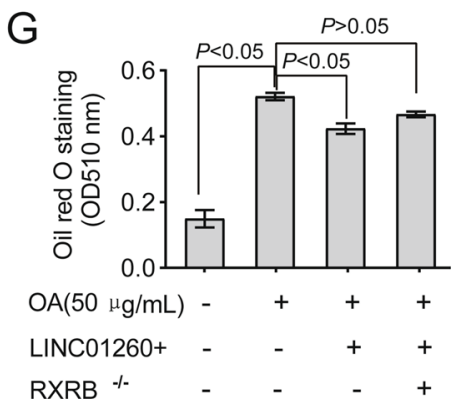

Fig. 5 (See legend on previous page.) 
translation, there are many regulators; any change could lead to different translation level. Also, for example, we detected FASN protein under LINC01260 overexpression, and found a decrease of FASN in LINC01260 overexpression group (in another work, data not shown here). It indicated that LINC01260 overexpression may influent hepatocytes steatosis from other mechanism. RXRB may not be the critical regulator related with LINC01260. Further efforts are needed to delineate the specific mechanisms.

It is undeniable that some limitations could be found for the present work. For example: (1) Oil red O staining is very generic for neutral lipid droplets. Only oleic acid, not other fatty acids (palmitic, palmitoleic, steric, or linoleic acid) were provided. Single component is hard to simulate in vivo state. Also, acute treatment of oleic acid for $48 \mathrm{~h}$ is different than chronic high fat conditions in vivo. Generally chronic high fat conditions in vivo are always accompanied with kinds of lipids changes, including saturated and unsaturated fatty acids. For example, in vivo high fat body usually includes high Triglyceride (TAG), Diglyceride (DAG), Phospholipids (PL), Phosphatidylethanolamine (PE), etc. It is the result of complex multi factor interaction, and single component is hard to simulate in vivo state. Our in vivo study in ongoing in another study.

(2) The present study investigated only steatosis as a biological endpoint, without investigation about cellular morphology (such as ballooning), inflammation or lipids content identification. The mechanism of NAFLD/NASH induced liver injury is considered to be a "two hit" phenomenon. The "first hit" includes inflammation and steotosis, which exacerbates liver sensitive to various "second hit" and lead to fibrosis [26]. Many studies proved that lipids plays critical function by inducing inflammation, cell injury and fibrosis [27]. Excessive lipid accumulation in liver is accompanied by series of histological changes from simple steatosis to non-alcoholic steatohepatitis, and will progress to cirrhosis with time [28]. Excessive lipid accumulation induced by the imbalance of liver lipid metabolism is the main cause of NAFLD. Additionally, more than 5\% hepatocytes with lipids are the histological standards in NAFLD diagnosis. Also, inflammation is one of important endpoints in our research team, and another ongoing research work is underway. Over all, we focused on hepatocytes steatosis in the present study.

Taken together, the present study provided the function of LINC01260 in the regulation of the lipid droplet formation process of NAFLD. As far as our knowledge, it is novel in reporting LINC01260 in lipids accumulation. Also, the present study indicated an informative read for the mechanism of LINC01260 in lipids. These in vitro findings indicated the clinical value of
LINC01260 in vivo, although largely keeps unknown about LINC01260 in vivo function. Overexpression of LINC01260 is of clinical value in NAFLD prevention and treatment.

\section{Conclusions}

In summary, we demonstrated that LINC01260 expression was significantly downregulated in the NAFLD cell model. Overexpression of LINC01260 inhibited the steatosis process induced by OA in liver cells. Collectively, our findings strongly suggest that LINC01260 may play an important role in regulating hepatic lipid metabolism through RXRB regulation and may serve as a potential therapeutic target for NAFLD.

\section{Abbreviations}

NAFLD: Nonalcoholic fatty liver disease; IncRNAs: Long noncoding RNAs; OA: Oleic acid; GOF: Gain-of-function; ceRNA: Competing endogenous RNA; qPCR: Quantitative real-time PCR.

\section{Supplementary Information}

The online version contains supplementary material available at https://doi. org/10.1186/s12986-021-00634-4.

Additional file 1. Table 1. Primer sequences for $\mathrm{qPCR}$.

\section{Acknowledgements}

RNA sequencing analysis was performed by KangChen Bio-tech, Shanghai, China.

Authors' contributions

SML designed the study; XXG and TS conducted the main experiments; WSM, YMZ, YQL, PG, DTZ, BYZ and PYW did sample analysis and data analysis, $X X G$, TS and SML wrote the manuscript; BYZ and PYW supported the study; SML, WSM and PG revised the paper. All authors read and approved the final manuscript.

\section{Funding}

This study was funded by the Cultivation Fund of National Natural Science Foundation of China in Shandong Provincial Qianfoshan Hospital (No. QYPY2020NSFC1004; QYPY2021NSFC0804), National Natural Science Foundation of China (No. 81400843), the Key Technology Research and Development Program of Shandong (No. 2017G006024) and Natural Science Foundation of Shandong Province (No. ZR2014HP033).

Availability of data and materials

All data generated or analyzed during this study are included in this published article or are available from the corresponding author on reasonable request.

\section{Declarations}

Ethics approval and consent to participate

This study was approved by the ethics committee of the Ethics Committee of Shandong University (No: [2017] S048).

Consent for publication

Not applicable.

Competing interests

The authors declare they have no competing interests. 


\section{Author details}

'Department of Laboratory Medicine, Shandong Provincial Qianfoshan Hospital, Shandong University, Jinan 250014, Shandong, People's Republic of China. ${ }^{2}$ Department of Laboratory Medicine, The First Affiliated Hospital of Shandong First Medical University, Jinan 250014, Shandong, People's Republic of China. ${ }^{3}$ Medical Research Center, The First Affiliated Hospital of Shandong First Medical University, Jinan 250014, Shandong, People's Republic of China. ${ }^{4}$ Blood Transfusion Department, Henan Provincial People's Hospital, People's Hospital of Zhengzhou University, Zhengzhou 450003, Henan, People's Republic of China. ${ }^{5}$ Department of Clinical Laboratory, The Affiliated Hospital of Shandong University of Traditional Chinese Medicine, Jinan 250014, Shandong, People's Republic of China.

\section{Received: 9 July 2021 Accepted: 30 November 2021}

Published online: 12 January 2022

\section{References}

1. Lindenmeyer CC, McCullough AJ. The natural history of nonalcoholic fatty liver disease: an evolving view. Clin Liver Dis. 2018;22:11-21.

2. Wang FS, Fan JG, Zhang Z, Gao B, Wang HY. The global burden of liver disease: the major impact of China. Hepatology. 2014;60:2099-108.

3. Quinn JJ, Chang HY. Unique features of long non-coding RNA biogenesis and function. Nat Rev Genet. 2016;17:47-62.

4. Sulaiman SA, Muhsin NIA, Jamal R. Regulatory non-coding RNAs network in non-alcoholic fatty liver disease. Front Physiol. 2019;10:279.

5. Yu F, Jiang Z, Chen B, Dong P, Zheng J. NEAT1 accelerates the progression of liver fibrosis via regulation of microRNA-122 and Kruppel-like factor 6. J Mol Med (Berl). 2017;95:1191-202.

6. Wang X. Down-regulation of IncRNA-NEAT1 alleviated the non-alcoholic fatty liver disease via mTOR/S6K1 signaling pathway. J Cell Biochem. 2018;119:1567-74.

7. Bian EB, Wang YY, Yang Y, Wu BM, Xu T, Meng XM, Huang C, Zhang L, Lv XW, Xiong ZG, Li J. Hotair facilitates hepatic stellate cells activation and fibrogenesis in the liver. Biochim Biophys Acta Mol Basis Dis. 2017; 1863:674-86.

8. Liu J, Tang T, Wang GD, Liu B. LncRNA-H19 promotes hepatic lipogenesis by directly regulating miR-130a/PPARY axis in non-alcoholic fatty liver disease. Biosci Rep. 2019, 39.

9. Wang H, Cao Y, Shu L, Zhu Y, Peng Q, Ran L, Wu J, Luo Y, Zuo G, Luo J, et al. Long non-coding RNA (IncRNA) H19 induces hepatic steatosis through activating MLXIPL and MTORC1 networks in hepatocytes. J Cell Mol Med. 2020;24:1399-412.

10. Ma M, Duan R, Shen L, Liu M, Ji Y, Zhou H, Li C, Liang T, Li X, Guo L. The IncRNA Gm15622 stimulates SREBP-1c expression and hepatic lipid accumulation by sponging the miR-742-3p in mice. J Lipid Res. 2020;61:1052-64.

11. Zhang Q, Wang J, Li H, Zhang Y, Chu X, Yang J, Li Y. LncRNA Gm12664-001 ameliorates nonalcoholic fatty liver through modulating miR-295-5p and CAV1 expression. Nutr Metab (Lond). 2020;17:13.

12. Yan C, Li J, Feng S, Li Y, Tan L. Long noncoding RNA Gomafu upregulates Foxo1 expression to promote hepatic insulin resistance by sponging miR139-5p. Cell Death Dis. 2018;9:289.

13. Salmena L, Poliseno L, Tay $Y$, Kats L, Pandolfi PP. A ceRNA hypothesis: the Rosetta Stone of a hidden RNA language? Cell. 2011;146:353-8.

14. Phelps M, Coss C, Wang H, Cook M: Registered report: Coding-independent regulation of the tumor suppressor PTEN by competing endogenous mRNAs. Elife. 2016; 5. pii: e12470. doi:https://doi.org/10.7554/eLife.12470.

15. Huang M, Zhong Z, Lv M, Shu J, Tian Q, Chen J. Comprehensive analysis of differentially expressed profiles of IncRNAs and circRNAs with associated co-expression and ceRNA networks in bladder carcinoma. Oncotarget. 2016;7:47186-200.

16. Li JH, Liu S, Zhou H, Qu LH, Yang JH. starBase v2.0: decoding miRNAceRNA, miRNA-ncRNA and protein-RNA interaction networks from largescale CLIP-Seq data. Nucleic Acids Res. 2014; 42:D92-D97.

17. Mikolasevic I, Milic S, Turk Wensveen T, Grgic I, Jakopcic I, Stimac D, Wensveen F, Orlic L. Nonalcoholic fatty liver disease: a multisystem disease? World J Gastroenterol. 2016;22:9488-505.

18. Diehl AM, Day C. Cause, pathogenesis, and treatment of nonalcoholic steatohepatitis. N Engl J Med. 2017;377:2063-72.
19. De Vincentis A, Rahmani Z, Muley M, Vespasiani-Gentilucci U, Ruggiero S, Zamani P, Jamialahmadi T, Sahebkar A. Long noncoding RNAs in nonalcoholic fatty liver disease and liver fibrosis: state-of-the-art and perspectives in diagnosis and treatment. Drug Discov Today. 2020;25:1277-86.

20. Chen Y, Huang H, Xu C, Yu C, Li Y. Long Non-Coding RNA profiling in a non-alcoholic fatty liver disease rodent model: new insight into pathogenesis. Int J Mol Sci 2017, 18.

21. Park TJ, Hwang MY, Moon S, Hwang JY, Go MJ, Kim BJ. Identification of a copy number variation on chromosome 20q13.12 associated with osteoporotic fractures in the Korean population. Genom Inform. 2016, $14: 216-221$.

22. Ma X, He Z, Li L, Yang D, Liu G. Expression profiles analysis of long noncoding RNAs identified novel IncRNA biomarkers with predictive value in outcome of cutaneous melanoma. Oncotarget. 2017;8:77761-70.

23. Wu DM, Han XR, Wen X, Wang S, Wang YJ, Shen M, Fan SH, Zhuang J, Zhang ZF, Shan Q, et al. Long non-coding RNA LINC01260 inhibits the proliferation, migration and invasion of spinal cord glioma cells by targeting CARD11 via the NF-kB signaling pathway. Cell Physiol Biochem. 2018;48:1563-78.

24. Choudhary NS, Kumar N, Duseja A. Peroxisome proliferator-activated receptors and their agonists in nonalcoholic fatty liver disease. J Clin Exp Hepatol. 2019;9:731-9.

25. Goto T. A review of the studies on food-derived factors which regulate energy metabolism via the modulation of lipid-sensing nuclear receptors. Biosci Biotechnol Biochem. 2019;83:579-88.

26. Abrams GA, Kunde SS, Lazenby AJ, Clements RH. Portal fibrosis and hepatic steatosis in morbidly obese subjects: a spectrum of nonalcoholic fatty liver disease. Hepatology. 2004;40:475-83.

27. Geng Y, Faber KN, Meijer V, Blokzijl H, Moshage H. How does hepatic lipid accumulation lead to lipotoxicity in non-alcoholic fatty liver disease? Hep Int. 2021;15:21-35.

28. Liu Q, Bengmark S, Shen Q. The role of hepatic fat accumulation in pathogenesis of non-alcoholic fatty liver disease (NAFLD). Lipids Health Dis. 2010;9:42-42.

\section{Publisher's Note}

Springer Nature remains neutral with regard to jurisdictional claims in published maps and institutional affiliations. 